\title{
ARTICLE \\ Protective Roles of Some Leafy and Non-leafy Vegetables against the Severity of Arsenic-induced Skin Lesions among Women Living in Rural Bangladesh: A Case Control Study
}

\author{
Shayla Nasrin ${ }^{1,2}$ Mahbuba Kawser $^{1 *}$ Selim Ahmed $^{3}$ Anup Kumar Saha $^{2}$ Anamul Haque ${ }^{2}$ \\ Rufaida Rahman $^{2}$ Akhi Akter ${ }^{1}$ Sheikh Nazrul Islam ${ }^{1}$ \\ 1. Institute of Nutrition and Food Science, University of Dhaka, Dhaka, 1000, Bangladesh \\ 2. Institute of child and mother health (ICMH), Matuail, Dhaka, 1362, Bangladesh \\ 3. School of Medicine, University Malaysia Sabah, Kotakinabalu Sabah, Malaysia
}

\section{ARTICLE INFO}

Article history

Received: 3 November 2021

Accepted: 17 December 2021

Published Online: 7 January 2021

Keywords:

Arsenic

Bangladesh

Leafy vegetables

Non-leafy vegetables

Skin lesion

Severity

\begin{abstract}
Arsenicosis is common among villagers as they drink more contaminatedwater since the arsenic-crisis in Bangladesh. Supplementation of vitamins and micronutrients in counteracting arsenic toxicity has been proved for arsenic treatment. This study was intended to assess protective and beneficial roles of some commonly eaten vegetables on the development and severity of arsenicinduced skin lesions. A case-control study among $(\mathrm{N}=122)$ adult rural-women (62 cases had various forms of arsenical skin-lesions e.g. melanosis/keratosis/ mixed-lesions and 60 sex-age-matched healthy-controls) was conducted in Shaharstee Upazilla of Chandpur district, Bangladesh. Socio-demographic data recorded in a pre-tested-questionnaire, 'per-day vegetables ingestion' of cases and controls were measured qualitative and quantitatively (24-hour recall-methods, food-frequency/week and food history-record/week). Multiple logistic regression/MLR analyses were performed to find out protective roles of some dietary leafy-vegetables/LVs and non-leafy vegetables/NLVs on arsenicosis and their influences on the degree of severity of arsenicosis also determined. Abstinence from taking some LVs/NLVs among cases than controls is associated with increased risk for arsenicosis $(\mathrm{P}<0.05)$. Amongst all most-frequently eaten vegetables $(\mathrm{n}=17)$ per day Momordica diocia has the highest skin protective role on arsenicosis [Adjusted odds ratio/AOR 8.2, 95\% CI (2.11-31.9), $\mathrm{P}=<0.01]$, followed by Ipomoea acquatica (AOR:7.3), Basella alba (AOR:6.2), Solanum tuberosum (AOR:4.0), Vigna unguiculata sesquipedalis (AOR:3.2), Trichosanthes anguina (AOR:1.2) and Abelmoschus esculentus (AOR:1.2). Moreover, severe skin lesion was observed as compared to non-severe cases (mild/moderate) for less intake frequencies of vegetables. This study outlined that commonly eaten vegetables have protective and beneficial roles on arsenic-induced skin lesions. Large samples longitudinal study of this important field of therapeutic-intervention is warranted.
\end{abstract}

\footnotetext{
*Corresponding Author:

Mahbuba Kawser,

Institute of Nutrition and Food Science, University of Dhaka, Dhaka, 1000, Bangladesh;

Email:mahbubakawser@gmail.com
}

DOI: https://doi.org/10.30564/jbr.v4i1.4050

Copyright (C) 2022 by the author(s). Published by Bilingual Publishing Co. This is an open access article under the Creative Commons Attribution-NonCommercial 4.0 International (CC BY-NC 4.0) License. (https://creativecommons.org/licenses/by-nc/4.0/). 


\section{Introduction}

Arsenic is a common environmental toxin. Exposure to arsenic (particularly its inorganic form) through contaminated food and drinking water is an important public health burden worldwide and is associated with increased risk of skin keratosis, neurotoxicity, congenital anomalies, and cancer ${ }^{[1]}$.

Bangladesh is the worst arsenic-affected country in the world and 'arsenic poisoning in drinking water' is her major public health problem especially among rural habitants since its first official disclosure in $1993^{[2-5]}$. Since then, Bangladesh has been fighting an un-equal battle due to lagging of sustainable surveillance of arsenicsafe water supply and effective arsenicosis management for arsenic-exposed populations as eighty percent of peoples live in villages and their daily consumption of arsenic contaminated water is also high $(>5$ liters per day) ${ }^{[5]}$. Study reported $12.6 \%$ of examined samples of water were arsenic contaminated in Bangladesh ${ }^{[6]}$. As arsenicosis case is diagnosed by only the presence of characteristic 'skin lesion', so in spite of having arseniccontaminated tube-well water, sub-clinical arsenic-induced health problem is being underreported ${ }^{[5]}$, although some improvements (increased awareness and less consumption of Arsenic-contaminated drinking water) cannot be denied ${ }^{[4]}$. World Health Organization (WHO) warned that few millions people of Bangladesh may suffer from arsenicinduced skin cancer over the next few decades if arsenicfree safe drinking water $(<10 \mu \mathrm{g} / \mathrm{L})$ is not ensured ${ }^{[7]}$.

Skin lesion is one of the most common sign of chronic arsenic exposure with arsenic concentration of $>100$ $\mu \mathrm{g} / \mathrm{L}$, which can occur within month or after several years of exposure and causes melanosis (early and common manifestation like hyperpigmentation, spotted pigmentation, depigmentation, and hypopigmentation), skin cancer (basal cell and squamous cell carcinoma/ Bowen's disease) and keratosis (advanced stage of arsenicosis), which especially appears on palm and sole in different manner as a late feature of arsenical-dermatosis, and later on transformed to squamous cell carcinoma ${ }^{[8]}$. It has been found that the risk of skin lesion did not decrease after reducing the exposure for up to several years ${ }^{[9]}$. Among the medical measures, remedial role of some foods, supplementation of vitamins and micronutrient in counteracting arsenic toxicity has been proved ${ }^{[5]}$. Medicinal plants have been used since prehistoric times to treat different types of diseases, due to the presence of bioactive components ${ }^{[10]}$. Both leafy and non-leafy vegetables have intrinsic active nutrients (especially antioxidant vitamin A, E and C, B-complex (B1, B2, B5,
B6, folic acid), linoleic acid, polysaccharides, calcium, manganese, magnesium, iron, and antioxidant minerals copper, zinc and selenium and abundant sources of bioactive plant's secondary metabolites like flavonoids and phenolic compounds (e.g. catechin, quercetin, Lectins, Pectins, and Glycosylated compounds) ${ }^{[1]}$.

Both phenolics and flavonoids have antioxidant, immune-modulatory, anti-proliferative and pro-apoptotic, antibacterial, anticancer properties ${ }^{[12]}$. Quercetin, an important flavonoid has immense free radical scavenging ability toward superoxide anions, peroxyl, and hydroxyl radicals, thus, reducing oxidative stress and inflammation and accelerated wound closure in cell scratch assay, reduced pro-inflammatory cytokine production, and immune cell infiltration, and help to regenerate the mucosal layer after injury ${ }^{[13-15]}$. Study ${ }^{[16]}$ reported dietary B-vitamins (particularly $\mathrm{B}_{1}, \mathrm{~B}_{6}, \mathrm{~B}_{12}$ ) intake is associated with lower urinary mono-methyl arsenic and oxidative stress marker among US adults. Moreover, a recent trial ${ }^{[1]}$ reported taking folic acid supplements, alone or in combination with other nutrients, might reduce blood arsenic and plasma homocysteine concentrations, potentially could facilitate arsenic methylation and excretion, thereby reducing arsenic toxicity in adults who had been exposed to arsenic-contaminated drinking water, compared to placebo. Previous study in Bangladesh reported arsenic induced skin lesions are more prevalent in men compared to women due to less efficient methylation of arsenic among men ${ }^{[17]}$. Study addressing roles of vegetable on arsenical skin-lesion is very scanty in Bangladesh and one decade earlier ${ }^{[18]}$ and showed diversified diet rich in 'gourds and root' vegetables reduced arsenic-induced skin lesion. To the best of our knowledge no study explicitly considered the protective roles of only vegetables (both leafy-vegetables/LVs and non-leafy vegetables/NLVs) against arsenic-associated skin lesions among rural women. Thus, understanding the importance of availability, higher intake rate of vegetables in rural areas, also as a source of cheapest nutrients, potential bioactive and nutraceuticals properties ${ }^{[3,12,19-}$ ${ }^{33}$, this study perceived to find out how protective roles of vegetables can play against the severity of arsenicinduced skin lesion. Findings of this study may assist understanding and addressing the remedial measure of higher-rate of arsenicosis among rural people and include proper steps at policy/or intervention-levels.

\section{Methodology}

\subsection{Study Population and Site}

'Source-population' of this 'case-control' study was all 
female population in nine villages out of three unions in Shaharastee Upazilla of Chandpur district, Bangladesh. Study place and population was selected according to the latest information obtained from the respective wing of the Directorate General of Health Services (DGHS), Bangladesh about the level of arsenic in drinking water. The report identified Shaharastee Upazilla as one of the worst affected area with arsenicosis ${ }^{[4]}$.

\subsection{Inclusion Criteria and Ethical Approval}

Every cases were randomly selected on the basis of defined criteria which were willing to participate in the study, married women living in the study area for 2 years/ or more, sources of drinking water must from tube wells, which at least once had been marked "red" by the DPHE person and having visible signs of skin lesions (hypo pigmentation/or melanosis, scaling/or keratosis and mixed lesions) attributable to arsenic in drinking water after 'clinical assessment'. Exclusion criteria were subject having skin lesions but not arsenic-induced/or having other serious diseases, whose drinking water source could not be ascertained properly and not willing to take part in the study.

Written consent was obtained from all participants according to Helsinki declaration before the beginning of the study and confidentiality was marinated for all subjects. The study proposal was reviewed and approved by the Ethical Board of the Institute of Child and Mother Health (ICMH), Matuail, Dhaka, Bangladesh.

\subsection{Sample Size Estimation and Collection of the Data}

By using a standard formula ${ }^{[34]}$ sample size per group (case/or control) was 58, and total sample size (both groups) was calculated $116 \approx 122$, considering expected proportion in controls $=0.05$, assumed odds ratio $=5.5$, confidence interval $(\mathrm{CI})=0.95$, and power $=0.8$. Thus 62 cases having arsenicosis and 60 sex-age-matched healthy controls without skin lesions (adult women $\geq 18$ years) were selected from the same study area. Control (non-cases samples of the source-population) was also confirmed clinically for not having arsenicosis. Cases were categorized according to the severity (mild/moderate or severe) of skin lesions.

\subsubsection{Socio-economic and Arsenic Related Data}

A pretested (pretested on $(\mathrm{n}=10)$ subjects were discarded) structured questionnaire was used to record the data (age, education, occupation, drinking/cooking water sources, duration and severity of arsenicosis) by direct interview of the cases and controls with same questionnaire, in the same manner. Three of the authors personally interviewed all participants (both cases and controls). Data were collected under strict supervision and collected data in the questionnaire were checked by day to day basis.

\subsubsection{Dietary-intake Information}

The study was aimed to determine whether there is any protective role of different dietary vegetables (LVs/NLVs) with severity and the development of arsenic-induced skin lesions. Both qualitative and quantitative approaches were applied to measure habitual dietary practices in terms of taking vegetables. Food consumption ${ }^{[35]}$ especially vegetables (both LV and NLVs) intake frequency per day at individual level was assessed for all 122 subjects (both case and control) by recording previous diet history, one24 hour food-recall, and food frequency questionnaire (FFQ) for a week with the aid of portion size display. All food intake frequencies and food data were coded using food conversion tables ${ }^{[36]}$ and then entered into computer for food analysis.

\subsection{Statistical Analysis}

Data (both socioeconomic and food intake) were analyzed by using Statistical Package (SPSS Inc, Chicago, IL, USA, version 23.0). Descriptive statistics were employed and values were expressed as frequency, percentage, mean and standard deviation as and where necessary. As total seventeen $(n=17)$ vegetables were frequently taken, simple logistic regressions (SLRs) have been used to examine possible associations between exposure (Daily Ingestion of leafy $(n=08) /$ non-leafy vegetables $(n=09)$ : Intake/or no-intake) and outcome (having arsenic-induced skin lesions: Yes/No). Multiple logistic regressions (MLR) were performed adjusting demographic variables, odds ratios (ORs) and $95 \%$ confidence-interval (CI) were obtained to identify potential roles of leafy/non-leafy vegetables ingestion (by comparing case and control) associated with arsenicinduced skin lesions. Among seventeen $(n=17)$ LVs and NLVs which were found statistically significant $(\mathrm{P}<0.10)$ in SLR were introduced in the MLR-analysis and the models were developed by 'Backward-StepwiseElimination. Hosmer-Lemeshaw goodness-of-fit and Nagelkarke-pseudo- $\mathrm{R}^{2}$ of the models were also observed. Moreover, cross-classification-analysis ( $\aleph^{2}$-test) have been used to examine possible associations of daily leafy/ or non-leafy vegetables intake in terms of severity of arsenic-induced skin lesions between severe and non- 
severe (mild/moderate) case-groups. P-value $<0.05$ was set as significance.

\section{Results}

\subsection{Background Characteristics, Pattern, Duration and Severity of Arsenicosis}

Mean ages (37.32 \pm 10.7 verses $35.73 \pm 9.7$ years) and occupation ( $90 \%$ housewives) of both groups e.g. cases (village women having arsenicosis) and control (agesex-matched healthy-controls without arsenicosis) are similar $(\mathrm{P}>0.05)$. Sources of drinking water for most of the cases $(83.9 \%)$ were Hand-pressed tube well and for most of the control (91.7\%) it was Pond/river/others sources. Consequently, who suffered from severe skin lesions $(n=33)$, all $(100 \%)$ used 'Hand-pressed tubewell' as drinking water sources, followed by $63.2 \%$ $(\mathrm{n}=12)$ and $70 \%(\mathrm{n}=07)$ used 'Hand-pressed tube-well' respectively by mild-skin-lesion and moderate-skinlesion sufferers. Majority (46.8\%) of the cases had rain drop lesions or white cluster (of them, $60.6 \%$ severe, $40 \%$ moderate and $26.3 \%$ had mild skin lesion) followed by Hypopigmentation (27.4\%) and Palmar keratosis (25.8\%). Vast majority (83.9\%) of cases had arsenicosis for $<4$ years $(1.2 \pm 0.5)$ and mostly $(82.3 \%)$ was diagnosed by doctor and NGO workers (6.1\%) (Table 1).

Table 1. Background characteristics of all participants and pattern, duration and severity of arsenic induced skin lesions among cases

\begin{tabular}{|c|c|c|c|c|c|}
\hline \multirow{3}{*}{ Characteristics } & \multicolumn{3}{|c|}{ Severity of arsenicosis of cases $n(\%)$} & \multirow{3}{*}{$\begin{array}{l}\text { Total cases } \\
(\mathrm{n}=62) \\
\text { n (\%) }\end{array}$} & \multirow{3}{*}{$\begin{array}{l}\text { Controls }(n=60) \\
n(\%)\end{array}$} \\
\hline & \multicolumn{2}{|c|}{ Not severe $(n=29)$} & \multirow[b]{2}{*}{ Severe $(n=33)$} & & \\
\hline & $\begin{array}{l}\text { Mild } \\
(\mathrm{n}=19)\end{array}$ & $\begin{array}{l}\text { Moderate } \\
(\mathrm{n}=10)\end{array}$ & & & \\
\hline \multicolumn{6}{|l|}{ Age } \\
\hline$\leq 20$ & $05(26.3)$ & $00(0.0)$ & $00(0.0)$ & $05(8.1)$ & $02(3.4)$ \\
\hline $21-30$ & $04(21.1)$ & $05(50.0)$ & $05(15.1)$ & $14(22.6)$ & $20(33.3)$ \\
\hline $31-40$ & $05(26.3)$ & $03(30.0)$ & $12(36.4)$ & $20(32.3)$ & $26(43.3)$ \\
\hline $41-50$ & $05(26.3)$ & $02(20.0)$ & $10(30.3)$ & $17(27.4)$ & $06(10.0)$ \\
\hline$\geq 51$ & $00(0.0)$ & $00(0.0)$ & $06(18.2)$ & $06(9.6)$ & $06(10.0)$ \\
\hline Mean \pm SD & $37.32 \pm 10.7^{*}$ & & & & $35.73 \pm 9.7^{*}$ \\
\hline \multicolumn{6}{|l|}{ Occupation } \\
\hline House wife & $19(100)$ & $09(90.0)$ & $29(87.9)$ & $57(91.9)^{* *}$ & $55(91.7)^{* *}$ \\
\hline Service & $00(00)$ & $01(10.0)$ & $04(12.1)$ & $05(8.1)$ & $05(8.3)$ \\
\hline \multicolumn{6}{|c|}{ Sources of drinking water } \\
\hline Hand-pressed tube-well & $12(63.2)$ & $07(70.0)$ & $33(100.0)$ & $52(83.9)$ & $11(18.3)$ \\
\hline Pond/river/others & $07(36.8)$ & $03(30.0)$ & $00(0.0)$ & $10(16.1)$ & $60(91.7)$ \\
\hline \multicolumn{6}{|l|}{ Patterns of skin lesion } \\
\hline $\begin{array}{l}\text { Rain drop lesions } \\
\text { (white cluster) }\end{array}$ & $05(26.3)$ & $04(40.0)$ & $20(60.6)$ & $29(46.8)$ & NA \\
\hline Hypopigmentation & $06(31.6)$ & $05(50.0)$ & $06(18.2)$ & $17(27.4)$ & \\
\hline Palmar keratosis & $08(42.1)$ & $01(10.0)$ & $07(21.2)$ & $16(25.8)$ & \\
\hline \multicolumn{6}{|c|}{ Duration (years) of arsenicosis } \\
\hline$<4$ & $14(73.6)$ & $08(80)$ & $30(90.9)$ & $52(83.9)$ & NA \\
\hline$\geq 4$ & $05(26.4)$ & $02(20)$ & $03(9.1)$ & $06(16.7)$ & \\
\hline Mean \pm SD & $1.2 \pm 0.5$ & & & & \\
\hline \multicolumn{6}{|l|}{ Diagnosed by } \\
\hline Doctor & $10(52.6)$ & $10(100)$ & $31(93.8)$ & $51(82.3)$ & NA \\
\hline NGO worker & $09(47.4)$ & $00(0.0)$ & $01(3.1)$ & $10(16.1)$ & \\
\hline Neighborhood & $00(0.0)$ & $00(0.0)$ & $01(3.1)$ & $01(1.6)$ & \\
\hline
\end{tabular}

${ }^{*}$ Student's T-test $\mathrm{P}>0.05^{* *}$ Chi-square test $\mathrm{P}>0.05$

$\mathrm{NA}=$ not applicable 


\subsection{Nutrient-rich Food Intake Per Day}

About $37.1 \%$ of the cases and almost half $(50.0 \%)$ of the controls ingested protein rich foods $(\mathrm{P}<0.05)$ per day. Lower consumption of protein-rich foods by cases was associated with severe arsenicosis while not severe (mild/ moderate) cases took higher rate of protein foods than severe group $(\mathrm{P}<0.05)$. Similarly, lower consumption of both LVs (38.7\%) and NLVs (37.1\%) among cases (both severe and not-severe) were observed than control $(\mathrm{LVs}=56.7 \%$ and $\mathrm{NLVs}=53.3 \%)$ (Table 2).

\subsection{Protective Role of Different Vegetables on Arsenic Induced Skin Lesions}

Table 3 outlined that among all $(n=17)$ LVs $(n=08)$ and NLVs $(\mathrm{n}=09)$ most of the vegetables have significant $(\mathrm{P}<0.05)$ protective roles on arsenic induced-skin lesions in unadjusted model/or bivariate analysis (shown by crude odds ratio/COR) except green aurum leaves, jute leaves and tomatoes $(\mathrm{P}>0.05)$. However, only seven vegetables (two LVs and five NLVs) remained in the final adjusted model (shown by adjusted odd ratios/AOR) of multiple logistic regression (MLR). MLR model showed amongst all most frequently eaten vegetables $(\mathrm{n}=17)$ per day, Momordica diocia (spiny gourd) has the highest skin protective role on arsenicosis [AOR 8.2 95\% CI (2.11-31.9), $\mathrm{P}=<0.01]$, followed by Ipomoea acquatica (Water spinach, AOR: 7.3), Basella alba (Indian spinach,
AOR:6.2), Solanum tuberosum (Potato, AOR:4.0), Vigna unguiculata sesquipedalis (Snake/long bean,AOR:3.2), Trichosanthes anguina (Snake gourd,AOR:1.2) snd Abelmoschus esculentus (Ladies finger, AOR:1.2). This study reported not taking LVs like Indian spinach (64.5\%) and water spinach (59.7\%) among cases than controls (23.3\% and $26.7 \%$ ) are respectively 6.2 and 7.3 -times (AOR) more likely to be associated with arsenicosis $(\mathrm{P}<0.01)$. Similarly, abstinence from taking some NLVs (e.g. spiny gourd, potato, snake gourd, long/snake bean and ladies finger) among cases than controls are respectively 8.2, 4.0, 1.2, 3.2 and 1.2-times (AORs) more likely to be associated with arsenicosis $(\mathrm{P}<0.05)$.

\subsection{Influence of the Intake of Different Vegetables on the Severity of Arsenicosis}

Table 4 showed severity of arsenicosis in terms of taking more/less vegetables between two groups (severe/ not severe) having arsenicosis. Lower consumption of vegetables (both LVs and NLVs) was associated with severe arsenicosis while not severe (mild/moderate) cases took higher rate of both LVs and NLVs than severe group $(\mathrm{P}<0.05)$. Among leafy vegetables those were frequently consumed per day by the study subjects, two LVs (Indian spinach or Basella alba and Water spinach or Ipomoea acquatica) reportedly showed to be protective against severe arsenicosis $(\mathrm{P}<0.05)$. Similarly, five NLVs (Spiny gourd or Momordica diocia, Potato or Solanum tuberosum,

Table 2. Nutrient-rich food intake frequency per day between groups

\begin{tabular}{|c|c|c|c|c|c|c|}
\hline \multirow{4}{*}{ Frequency distribution of food intake } & \multirow{4}{*}{$\begin{array}{l}\text { Total } \\
\text { Cases } \\
(n=62)\end{array}$} & \multicolumn{3}{|c|}{ Case n (\%) } & \multirow[t]{4}{*}{ Control } & \multirow{4}{*}{ Test- statistic } \\
\hline & & \multicolumn{3}{|c|}{ Classification of arsenicosis } & & \\
\hline & & \multicolumn{2}{|c|}{ Not severe $(n=29)$} & \multirow{2}{*}{$\begin{array}{l}\text { Severe } \\
(\mathrm{n}=\mathbf{3 3})\end{array}$} & & \\
\hline & & $\begin{array}{l}\text { Mild } \\
(n=19)\end{array}$ & $\begin{array}{l}\text { Moderate } \\
(\mathrm{n}=10)\end{array}$ & & & \\
\hline \multicolumn{7}{|l|}{ Animal-protein-rich foods } \\
\hline Yes & $23(37.1)$ & $10(52.6)$ & $05(50.0)$ & $09(27.3)$ & $30(50.0)$ & \multirow{3}{*}{$\mathbf{P}^{*}$} \\
\hline No & $39(62.9)$ & $09(47.4)$ & $05(50.0)$ & $16(72.7)$ & $30(50.0)$ & \\
\hline P-value & $\mathrm{P}^{*}$ & & & & & \\
\hline \multicolumn{7}{|l|}{ Mineral and vitamin-rich foods } \\
\hline \multicolumn{7}{|l|}{ Leafy vegetable/LVs $(n=08)$} \\
\hline Yes & $24(38.7)$ & $07(21.1)$ & $05(40.0)$ & $12(54.5)$ & $34(56.7)$ & \multirow{2}{*}{$\mathrm{P}^{*}$} \\
\hline No & $38(61.3)$ & $12(78.9)$ & $05(80.0)$ & $15(45.5)$ & $26(43.3)$ & \\
\hline P-value & $\mathrm{P}^{*}$ & & & & & \\
\hline \multicolumn{7}{|l|}{ Non-leafy vegetables/NLVs $(n=09)$} \\
\hline Yes & $23(37.1)$ & $08(42.1)$ & $04(40.0)$ & $11(33.3)$ & $32(53.3)$ & \multirow{2}{*}{$\mathrm{P}^{*}$} \\
\hline No & $41(62.9)$ & $11(57.9)$ & $06(60.0)$ & $22(66.7)$ & $28(46.7)$ & \\
\hline P-value & $\mathrm{P}^{*}$ & & & & & \\
\hline
\end{tabular}

${ }^{*}$ Chi-square/Fisher's Exact test and $\mathrm{P}<0.05$ 
Table 3. Protective role of different vegetables on arsenic induced skin lesions

\begin{tabular}{|c|c|c|c|c|c|c|}
\hline Leafy vegetables & & Case $(n=62)$ & $\begin{array}{l}\text { Control } \\
(n=60)\end{array}$ & $\begin{array}{l}\text { COR } \\
(95 \% \text { CI })\end{array}$ & (B) & $\begin{array}{l}\text { AOR } \\
(95 \% \text { CI })\end{array}$ \\
\hline \multirow{2}{*}{$\begin{array}{l}\text { Basella alba } \\
\text { (Indian spinach) }\end{array}$} & Intake $^{R}$ & $22(35.5)$ & $46(76.7)$ & 1 & & \\
\hline & No & $40(64.5)$ & $14(23.3)$ & $4.5(1.7-12.2)^{* *}$ & $\mathrm{~B}=1.839$ & $6.2(1.58-24.9)^{* *}$ \\
\hline \multirow{2}{*}{$\begin{array}{l}\text { Amaranthus tricolor } \\
\text { (Joseph's coat) }\end{array}$} & Intake $^{R}$ & $04(6.5)$ & $06(10.0)$ & 1 & & \\
\hline & No & $58(93.5)$ & $54(90.0)$ & $2.39(7.53-7.5)^{*}$ & & \\
\hline \multirow{2}{*}{$\begin{array}{l}\text { Amaranthus dubius } \\
\text { (Amaranth) }\end{array}$} & Intake $^{\mathrm{R}}$ & $06(9.7)$ & $04(6.7)$ & 1 & & \\
\hline & No & $56(90.3)$ & $56(93.3)$ & $2.28(.918-5.7)^{*}$ & & \\
\hline \multirow{2}{*}{$\begin{array}{l}\text { Cucurbita maxima } \\
\text { (Pumpkin leaves) }\end{array}$} & Intake $^{\mathrm{R}}$ & $32(51.5)$ & $24(40.0)$ & 1 & & \\
\hline & No & $30(48.5)$ & $36(60.0)$ & $2.00(.80-4.9)^{*}$ & & \\
\hline \multirow{2}{*}{$\begin{array}{l}\text { Ipomoea acquatica } \\
\text { (Water spinach) }\end{array}$} & Intake $^{\mathrm{R}}$ & $25(40.3)$ & $50(73.3)$ & 1 & & \\
\hline & No & $37(59.7)$ & $10(26.7)$ & $4.1(1.6-10.6)^{* *}$ & $\mathrm{~B}=1.984$ & $7.3(1.91-27.6)^{* * *}$ \\
\hline \multirow{2}{*}{$\begin{array}{l}\text { Colocasia antiquorum } \\
\text { (green aurum leaves) }\end{array}$} & Intake $^{\mathrm{R}}$ & $29(46.7)$ & $26(43.3)$ & 1 & & \\
\hline & No & $33(53.3)$ & $34(56.7)$ & $1.82(.75-4.4)$ & & \\
\hline \multirow{2}{*}{$\begin{array}{l}\text { Nasturtium officinale } \\
\text { (Water cress) }\end{array}$} & Intake $^{\mathrm{R}}$ & $04(6.5)$ & $16(13.3)$ & 1 & & \\
\hline & No & $58(93.5)$ & $52(86.7)$ & $2.14(.73-6.3)^{*}$ & & \\
\hline \multirow{2}{*}{$\begin{array}{l}\text { Corchorus capsularis } \\
\text { (Jute leaves) }\end{array}$} & Intake $^{\mathrm{R}}$ & $23(37.1)$ & $18(30.0)$ & 1 & & \\
\hline & No & $39(62.9)$ & $42(70.0)$ & $1.62(.66-3.9)$ & & \\
\hline \multicolumn{7}{|l|}{ Non-Leafy vegetables } \\
\hline \multirow{2}{*}{$\begin{array}{l}\text { Cucurbita maxima } \\
\text { (Pumpkin) }\end{array}$} & Intake $^{\mathrm{R}}$ & $37(59.7)$ & $46(76.6)$ & 1 & & \\
\hline & No & $25(50.3)$ & $14(23.4)$ & $2.22(.83-5.95)^{*}$ & & \\
\hline \multirow{2}{*}{$\begin{array}{l}\text { Momordica charantia } \\
\text { (Bitter gourd) }\end{array}$} & Intake $^{\mathrm{R}}$ & $29(46.8)$ & $38(63.3)$ & 1 & & \\
\hline & No & $33(53.2)$ & $22(36.7)$ & $1.96(.80-4.8)^{*}$ & & \\
\hline \multirow{2}{*}{ Solanum lycopersicum (Tomato) } & Intake $^{R}$ & 08 (12.9) & $10(16.6)$ & 1 & & \\
\hline & No & $54(87.1)$ & $50(83.4)$ & $1.17(.36-3.9)$ & & \\
\hline \multirow{2}{*}{$\begin{array}{l}\text { Momordica diocia } \\
\text { (Spiny gourd) }\end{array}$} & Intake $^{\mathrm{R}}$ & $24(38.7)$ & $34(56.7)$ & 1 & & \\
\hline & No & $38(61.3)$ & $26(43.3)$ & $4.48(1.76-11.4)^{* *}$ & $\mathrm{~B}=2.107$ & $8.2(2.11-31.9)^{* *}$ \\
\hline \multirow{2}{*}{$\begin{array}{l}\text { Daucus carota } \\
\text { (Carrot) }\end{array}$} & Intake $^{R}$ & $03(4.8)$ & $08(13.3)$ & 1 & & \\
\hline & No & $59(95.2)$ & $52(86.7)$ & $3.03(.63-14.5)^{*}$ & & \\
\hline \multirow{2}{*}{$\begin{array}{l}\text { Solanum tuberosum } \\
\text { (Potato) }\end{array}$} & Intake $^{\mathrm{R}}$ & $37(59.7)$ & $52(86.7)$ & 1 & & \\
\hline & No & $25(40.3)$ & $08(13.3)$ & $4.22(1.67-10.6)^{* * *}$ & $\mathrm{~B}=1.404$ & $4.0(1.12-14.7)^{*}$ \\
\hline \multirow{2}{*}{$\begin{array}{l}\text { Vigna unguiculata sesquipedalis } \\
\text { (Snake/long bean) }\end{array}$} & Intake $^{\mathrm{R}}$ & $23(37.1)$ & $42(70.0)$ & 1 & & \\
\hline & No & $39(62.9)$ & $18(30.0)$ & $3.08(1.15-8.2)^{*}$ & $\mathrm{~B}=1.177$ & $3.2(.87-12.1)^{*}$ \\
\hline \multirow[t]{2}{*}{$\begin{array}{l}\text { Trichosanthes anguina } \\
\text { (Snake gourd) }\end{array}$} & Intake $^{R}$ & $24(38.7)$ & $54(90.0)$ & 1 & & \\
\hline & No & $38(61.3)$ & $06(10.0)$ & $3.29(1.31-8.2)^{*}$ & $\mathrm{~B}=.197$ & $1.2(.22-6.7)^{*}$ \\
\hline \multirow{2}{*}{$\begin{array}{l}\text { Abelmoschus esculentus (Ladies } \\
\text { finger) }\end{array}$} & Intake $^{\mathrm{R}}$ & $33(53.2)$ & $50(83.3)$ & 1 & & \\
\hline & No & $29(46.8)$ & $10(16.7)$ & $2.38(.97-5.8)^{*}$ & $\mathrm{~B}=.222$ & $1.2(.27-5.9)^{*}$ \\
\hline
\end{tabular}

Overall percentage $81.5 \%,-2 \mathrm{LL}=71.425$, Nagelkerke $\mathrm{R}$ Square $=.537$, Model co-efficient (Chi-square) $=44.748$, degree of freedom $/$ $\mathrm{df}=5, \mathrm{P}=0.000$, Hosmer Lemeshow goodness of fit $=5.716, \mathrm{P}=.679$, Crude odds ratio $=\mathrm{COR}$, Beta coefficient $=\mathrm{B}$, Adjusted Odds ratio $=\mathrm{AOR},{ }^{\mathrm{R}}$ Reference category, ${ }^{*} \mathrm{P}<0.05,{ }^{* *} \mathrm{P}<0.01$ 
Snake/long bean or Vigna unguiculata sesquipedalis, Snake gourd or Trichosanthes anguina and ladies finger or Abelmoschus esculentus) have been showed protective effect against the development of severe arsenic-induced skin lesion $(\mathrm{P}<0.05)$.

\section{Discussion}

This study showed protective roles of some leafy and non-leafy vegetables on arsenic-induced skin lesion among women in rural Bangladesh and less intakefrequency of these vegetables also reported to influence on the severity of the skin lesion. The positive association of some leafy and non-leafy vegetables with the extent of arsenic related skin lesions can be explained by the facts that vegetables are one of the richest natural sources of vitamins (especially antioxidant vitamin A, C, E, and B-complex) and minerals (iron and antioxidant copper, zinc, and selenium) which have concomitant roles of both essential nutritive activities and anti-oxidants capacity, have been shown to exert protective effects on bodyburden of arsenic. Previous study ${ }^{[18]}$ also reported intake of different gourds, roots (NLVs) and protein inversely

Table 4. Influence of the intake of different vegetables on the severity of arsenicosis

\begin{tabular}{|c|c|c|c|c|c|}
\hline \multirow[t]{2}{*}{ Leafy vegetables } & & \multirow{2}{*}{$\begin{array}{l}\begin{array}{l}\text { Total } \\
(\mathrm{n}=62)\end{array} \\
\mathrm{n}(\%)\end{array}$} & \multicolumn{3}{|c|}{ Having skin lesions n (\%) } \\
\hline & & & $\begin{array}{c}\text { Severe } \\
(\mathrm{n}=\mathbf{3 3})\end{array}$ & $\begin{array}{l}\text { Not severe } \\
(n=29)\end{array}$ & P-value $^{(a)}$ \\
\hline \multirow[t]{2}{*}{ Indian spinach } & Intake & $41(66.1)$ & $14(42.4)$ & $27(93.1)$ & $\mathrm{P}=0.005^{* *}$ \\
\hline & No & $21(33.9)$ & $19(57.6)$ & $02(06.9)$ & \\
\hline \multirow[t]{2}{*}{ Joseph's coat } & Intake & $04(6.5)$ & $01(3.0)$ & $03(10.3)$ & $\mathrm{P}=0.332$ \\
\hline & No & $58(93.5)$ & $32(97.0)$ & $26(89.7)$ & \\
\hline \multirow[t]{2}{*}{ Amaranth } & Intake & $06(9.7)$ & $01(3.0)$ & $05(17.2)$ & $\mathrm{P}=0.089$ \\
\hline & No & $56(90.3)$ & $32(97.0)$ & $24(82.8)$ & \\
\hline \multirow[t]{2}{*}{ Pumpkin leaves } & Intake & $32(51.5)$ & $17(51.5)$ & $15(51.7)$ & $\mathrm{P}=0.987$ \\
\hline & No & $30(48.5)$ & $16(48.5)$ & $14(48.3)$ & \\
\hline \multirow[t]{2}{*}{ Water spinach } & Intake & $25(40.3)$ & $09(27.3)$ & $16(55.2)$ & $\mathrm{P}=0.025^{*}$ \\
\hline & No & $37(59.7)$ & $24(72.7)$ & $13(44.8)$ & \\
\hline \multirow[t]{2}{*}{ Green aurum leaves } & Intake & $29(46.8)$ & $17(51.5)$ & $12(41.4)$ & $\mathrm{P}=0.425$ \\
\hline & No & $33(53.2)$ & $16(8.5)$ & $17(58.6)$ & \\
\hline \multirow[t]{2}{*}{ Water cress } & Intake & $04(6.5)$ & $03(9.1)$ & $01(3.4)$ & $\mathrm{P}=0.616$ \\
\hline & No & $58(93.5)$ & $30(90.9)$ & $28(96.6)$ & \\
\hline \multirow[t]{2}{*}{ Jute leaves } & Intake & $23(37.1)$ & $10(30.3)$ & $13(44.8)$ & $\mathrm{P}=0.237$ \\
\hline & No & $39(62.9)$ & $13(69.7)$ & $16(55.2)$ & \\
\hline \multicolumn{6}{|l|}{ Non-leafy vegetables } \\
\hline \multirow[t]{2}{*}{ Pumpkin } & Intake & 37 (59.7) & $18(54.5)$ & $19(65.5)$ & $\mathrm{P}=0.380$ \\
\hline & No & $25(40.3)$ & $15(45.5)$ & $10(34.5)$ & \\
\hline \multirow[t]{2}{*}{ Bitter gourd } & Intake & $29(46.8)$ & $13(39.4)$ & $16(55.2)$ & $\mathrm{P}=0.214$ \\
\hline & No & $33(55.2)$ & $20(60.6)$ & $13(44.8)$ & \\
\hline \multirow[t]{2}{*}{ Tomato } & Intake & 08 (12.9) & $03(9.1)$ & $05(17.2)$ & $\mathrm{P}=0.456$ \\
\hline & No & $54(87.1)$ & $30(90.9)$ & $24(82.8)$ & \\
\hline \multirow[t]{2}{*}{ Spiny gourd } & Intake & $24(38.7)$ & $06(18.2)$ & $18(62.1)$ & $\mathrm{P}=0.004^{* * *}$ \\
\hline & No & $38(61.3)$ & $27(81.8)$ & $11(37.9)$ & \\
\hline \multirow[t]{2}{*}{ Carrot } & Intake & $03(4.8)$ & $00(0.0)$ & $03(10.3)$ & $\mathrm{P}=0.097$ \\
\hline & No & $59(95.2)$ & $33(100.0)$ & $26(89.7)$ & \\
\hline \multirow[t]{2}{*}{ Potato } & Intake & $37(59.7)$ & $14(42.4)$ & $23(79.3)$ & $\mathrm{P}=0.003^{* * *}$ \\
\hline & No & $25(40.3)$ & $19(57.6)$ & $06(20.7)$ & \\
\hline \multirow[t]{2}{*}{ Snake/long/Asparagus bean } & Intake & $23(37.1)$ & $07(21.2)$ & $16(55.2)$ & $\mathrm{P}=0.006^{* * *}$ \\
\hline & No & $39(62.9)$ & $26(78.8)$ & $13(44.8)$ & \\
\hline \multirow[t]{2}{*}{ Snake gourd } & Intake & $24(38.7)$ & $08(24.2)$ & $16(55.2)$ & $\mathrm{P}=0.013^{*}$ \\
\hline & No & $38(61.3)$ & $25(75.8)$ & $13(44.8)$ & \\
\hline \multirow[t]{2}{*}{ Ladies finger } & Intake & $33(53.2)$ & $12(36.4)$ & $21(72.4)$ & $\mathrm{P}=0.005^{* *}$ \\
\hline & No & $29(46.8)$ & $21(63.6)$ & $08(27.6)$ & \\
\hline
\end{tabular}

${ }^{\circledR}$ Chi-square test/Fisher's Exact Test; Not severe=mild/moderate arsenicosis; ${ }^{*} \mathrm{P}<0.05,{ }^{* * *} \mathrm{P}<0.01$ 
associated with arsenic-induced skin lesion risk among male and female cohort in Bangladesh.

Abstinence from the dietary intake of leafy/non-leafy vegetables among cases than controls is $(\mathrm{P}<0.05)$ more likely to be associated with arsenicosis. On the other hand, Consuming more leafy vegetables like 'Indian spinach (Basella alba)' and 'water spinach (Ipomoea acquatica)' and non-leafy vegetables like 'spiny gourd (Momordica diocia)', 'Potato (Solanum tuberosum)', 'snake gourd (Trichosanthes anguina)', 'snake/long bean (Vigna unguiculata sesquipedalis)' and 'Ladies finger (Abelmoschus esculentus)' were found to have less severe arsenic-induced skin lesions (mild/moderate) than severe one. This may be due to having intrinsic nutrient potentials and presence of other non-nutritive bioactive compounds and phytochemical characteristics of different leafy and non-leafy vegetables as reported by the most of the prior and recent studies ${ }^{[1,12,19-26,27-31,33,37]}$. As mentioned earlier vegetables are important for human nutrition in terms of bioactive nutrient molecules (dietary fiber, antioxidant pro-vitamin A/or Carotenoids, vitamin $\mathrm{C}$, vitamin $\mathrm{E}$ and minerals e.g. copper, zinc, selenium) and non-nutritive phytochemicals (phenolic compounds, flavonoids, saponin, bioactive peptides, glycoside etc.). These nutrient and non-nutrient molecules reduce the risk of non-communicable diseases ${ }^{[11,37]}$. Medicinal plants $[9,10,19-21,33,38]$ those having substantial amount of nutrients and non-nutrient poly-phenolic components (plant's secondary metabolites) have potent abilities to act as antioxidant, antibacterial and anti-inflammatory agents which accelerate the rapid healing of damaged skin tissue at various stages of wound healing (i.e., collagenation, wound closure and epithelialization) in addition to reducing damage caused by oxygen radicals in the area of dermal injury ${ }^{[19]}$. Moreover, antioxidant fights against free radicals and exert their action either by scavenging the reactive oxygen species or protecting the oxidant defense mechanism. Thus, presence of different nutrients and non-nutrients in LVs and NLVs would offer a synergistic effects to ensure the protection against arsenic-induced skin lesion among women.

In this study, dietary intake of two LVs (B. alba and I. acquatica) were found to be negatively associated with arsenicosis and not consuming these two LVs, the probability of infecting arsenic-induced skin lesion increased respectively 6.2 and 7.3 -folds among cases than controls $(\mathrm{P}<0.01)$. This might be due to arsenic is excreted following methylation reactions, which are mediated by folate ${ }^{[1]}$ and LVs are excellent, richest source of folate, thus folic acid with other bioactive polyphenols could facilitate arsenic methylation and excretion, performed antioxidant and free radical scavenging activity thereby reducing arsenic toxicity and skin lesions. Studies reported B. alba has great ethno-medicinal and nutritional importance as the plant is rich in vitamin A (carotenoids), vitamin $\mathrm{C}$, organic acids and many amino acids along with active constituents like basella saponin, kaempherol, betalain, flavonoids, saponins, peptide and phenolic compounds which are therapeutically used for antioxidant, anti-inflammatory, antiulcer, antifungal, anticonvulsant, analgesic activities and for the treatment of skin diseases and many more ${ }^{[22,26,27,30]}$. Similarly, higher intake $(93.1 \%)$ of $I$. acquatica by mild/moderate arsenicosis group than that of severe (42.4\%) was significantly associated with the degree of severity of arsenicosis. This might be due to the prophylactic effect of ascorbic acid ${ }^{[39]}$ and antiapoptotic potential of both phenolic and flavonoids against arsenic-toxicity through antioxidant mechanism ${ }^{[23,25,31]}$. Studies reported abundant presence of different nutrients (e.g. ß-carotenes and ascorbic acid), and nonnutrients (phenolics: gallic acid and chlorogenic acid; flavonoids compounds: myricetin, quercetin and apigenin) in I. aquatica would play crucial role in overall protective and cumulative effect against arsnic-induced skin lesion ${ }^{[23,31]}$. Moreover, study reported phenolics and saponins exhibited chelating properties to mop up arsenites from the body ${ }^{[25]}$.

This study showed dietary intake of Momordica diocia (spiny gourd) has $88 \%$ (inverting odds ratio 8.2 ) averting role in occurring arsenicosis which means controls are less likely to develop arsenicosis than cases due to more ingestion of spiny gourd and has the highest skin protective role among all vegetables, followed by other NLVs like Solanum tuberosum (75\%), Vigna unguiculata sesquipedalis (69\%), Trichosanthes anguina (17\%) and Abelmoschus esculentus (17\%). This might be due to the nutrient contents of ascorbic acid, the ratio of unsaturated fatty acid of spiny gourd ${ }^{[29]}$, and antioxidant, antibacterial, anti-inflammatory, antimicrobial and anti-allergic agents of most of the medicinal plants due to possessing bioactive compounds and the phytochemical substances (poly phenols) as reported most of the studies ${ }^{[19-21,33]}$. Study on animal model also reported aqueous extract of spiny gourd flesh might have suppressive effect, therapeutic potential for allergic responses (by inhibiting histamine release and decreased IgE levels) in vitro and in vivo as compared to control and alleviated development of atopic dermatitis-like skin lesions ${ }^{[29]}$. Additionally, considering the diversity of the phyto-constituents and bioactive metabolites (gallic acid, chlorogenic acid, polyphenols, flavonoids like Rutin, alkaloids like Solanine) present in $S$. tuberosum, studies reported to promote the wound healing 
process by increasing epithelialization rate and collagen synthesis and subsequent decreasing the overproduction of free radicals, facilitating oxygen diffusion mainly due to potatoes antioxidant, antibacterial capabilities. Thus, studies reported varieties of $S$. tubersome contain anthocyanin which suppress the modulation of atopic dermatitis ${ }^{[40]}$ and can heal wound in experimental mice ${ }^{[24]}$.

Studies outlined high nutritional contents (proteins, carbohydrates, calories, dietary fiber trace elements and macro-minerals) and presence of different bioactive compounds (polyols, phytosterols, phenolics, neochlorogenic acid, chlorogenic acid, caffeic acids and saponins) in cowpea/asparagus/snake bean's seeds and pods ${ }^{[41-44]}$. Thus having rich contents of both nutrient and non-nutrient substances, in this study snake bean ( $V$. unguiculata sesquipedalis) showed protective role against arsenic-induced skin lesion through performing its antioxidant (radical scavenging activity), antibacterial, anti-inflammatory, and anti-allergic activities ${ }^{[42-44]}$. Moreover, another NLV snake gourd (T. anguina) is a good source of Vitamin A, Vitamin $\mathrm{B}$ (mainly niacin), Vitamin $\mathrm{C}$ and minerals (magnesium, calcium, potassium, phosphorus, iron). The plant is richly constituted with a series of chemical constituents like flavonoids, carotenoids, glycosides, saponins phenolic acids which makes the plant pharmacologically and therapeutically active ${ }^{[32,45]}$.

Like this study, okra (Abelmoschus Esculentus) reported to have potential dietary medicine with nutraceutical importance against gastric lesions in mice model ${ }^{[28]}$. Okra has intrinsic active nutritional values (Vitamin A, K and C, B-vitamins (B1, B2, B6, folic acid), linoleic acid, polysaccharides, calcium, iron, manganese, and magnesium) along with huge nutraceutical (antioxidant, immunomodulatory, Antiproliferative and proapoptotic) and therapeutic potentials (antibacterial, anticancer, antidiabetic, lipid lowering agents) for having bioactive components (phytochemicals e.g. poly phenols like catechin, flavonoids (quercetin), Lectins, Pectins, Glycosylated compounds) ${ }^{[12,14,28,46,47]}$.

\section{Conclusions}

This study showed the protective and beneficial roles of some commonly eaten vegetables against both development and severity of arsenic-induced skin lesions. Large samples longitudinal study in this important field of therapeutic intervention is warranted.

\section{Strength and limitation of the study}

This case-control was conducted to assess the role of dietary intake of some 'vegetables' on arsenic- induced skin-lesions. Both qualitative and quantitative approaches (one 24-hour recall, food frequency/week and food history-record/week) were applied to measure habitual dietary practices in terms of taking leafy and non-leafy vegetables. Multiple logistic regression (MLR) analyses were performed to find out protective roles of some leafy $(\mathrm{n}=08)$ and non-leafy $(\mathrm{n}=09)$ vegetables on arsenic induced skin-lesions (arsenicosis) among rural women while adjusting demographic variables. Moreover, how dietary intake frequencies of vegetables influence the degree of severity on arsenicosis among village women also determined. However, recall bias- the main limitation of case-control study cannot be ruled out, and lack of information on sources of water for irrigation and arsenic contents of foods of those particular areas and not including males in the study- were other limitations. Despite some limitations this study has distinct strengths, most of the studies performed using animal model to see the phytochemical properties of plants, or association of nutrients with non-communicable diseases (NCDs), only few studies have evaluated the impact of vegetables on arsenic-associated skin lesion. In particular, this study helps to fill an important research gap to find out the strength of relationship about the protective roles of some leafy and non-leafy vegetables with the arsenic induced skin-lesions.

\section{Author Contributions}

Nasrin S, Islam SN, Kawser M, and Ahmed S conceptualized the Study and Islam SN was the lead supervisor, field supervisions and medical supports were provided by Saha AK and Haque A while field supervisions at different stages were accomplished by Rahman R and Akter A. Methodology was developed by Nasrin S, Islam SN and Kawser M. Field training given to the staffs and acquisition of data by Nasrin S, Rahman R and Akter A. Statistical data were analyzed and interpreted by Kawser M. Literature Reviewed by Kawser M, Rahman R, Akter A and Nasrin S. Nasrin S and Kawser $\mathrm{M}$ prepared the original draft of the manuscript. Islam SN, Ahmed S, Saha AK and Haque A had significant contribution for critical intellectual contents. All authors read, revised, edited and approved the final manuscript.

\section{Conflicts of Interest}

The authors declare no potential conflicts of interest regarding the publication of this paper.

\section{Funding}

This research did not receive any specific grant from 
funding agencies in the public, commercial, or not-forprofit sectors.

\section{Acknowledgement}

Authors are grateful to all participants of this study for their patience and co-operation during the dietary data collection and also thankful to all collaborative staffs for its accomplishment. Authors are in-debt to Mr. Shah Muhammad Anayetullah Siddiqui, principle scientific officer at Institute of Nutrition and Food Science (INFS), University of Dhaka, Bangladesh, for his co-operation and supervision in dietary data collection.

\section{References}

[1] Bae, S., Kamynina, E., Guetterman, H.M., et al., 2021. Provision of folic acid for reducing arsenic toxicity in arsenic-exposed children and adults. Cochrane Database Syst Rev. 2021(10).

DOI: https://doi.org/10.1002/14651858.CD012649. pub2.

[2] Choudhury, M.I.M., Shabnam, N., Ahsan, T., et al., 2018. Cutaneous Malignancy due to Arsenicosis in Bangladesh: 12-Year Study in Tertiary Level Hospital. Biomed Res Int.

DOI: https://doi.org/10.1155/2018/4678362.

[3] Chowdhury, W.K., Tisha, A., Akter, S., et al., 2017. The Role of Arsenic on Skin Diseases, Hair Fall and Inflammation: An Immunological Review and Case Studies. J Clin Exp Dermatol Res. 08(02). DOI: https://doi.org/10.4172/2155-9554.1000384.

[4] Chakraborti, D., Rahman, M.M., Mukherjee, A., et al., 2015. Groundwater arsenic contamination in Bangladesh-21 Years of research. J Trace Elem Med Biol. 31, 237-248.

DOI: https://doi.org/10.1016/j.jtemb.2015.01.003.

[5] Ahmad, S.A., Khan, M.H., Haque, M., 2018. Arsenic contamination in groundwater in bangladesh: Implications and challenges for healthcare policy. Risk Manag Healthc Policy. 11, 251-261.

DOI: https://doi.org/10.2147/RMHP.S153188.

[6] BBS and UNICEF Bangladesh Multiple Indicator Cluster Survey 2012-2013, ProgotirPathey: Final Report. Bangladesh Bureau of Statistics (BBS) and UNICEF Bangladesh, 2014, Dhaka, Bangladesh. Published online 2014. [Accessed October, 2021]

[7] World Health Organization, 2018. Arsenic in Drinking-Water, Background Document for Development of WHO Guidelines for Drinking-Water Quality. [Accessed 2021, October]

[8] Das, N.K., Sengupta, S.R., July 2014. Arsenicosis :
Diagnosis and treatment Seminar: Chronic Arsenicosis in India Arsenicosis : Diagnosis and treatment. DOI: https://doi.org/10.4103/0378-6323.45098.

[9] Argos, M., Kalra, T., Pierce, B.L., et al., 2011. A prospective study of arsenic exposure from drinking water and incidence of skin lesions in Bangladesh. Am J Epidemiol. 174(2), 185-194.

DOI: https://doi.org/10.1093/aje/kwr062.

[10] Inoue, M., Hayashi, S., Craker, L.E., 2018. Role of Medicinal and Aromatic Plants, Past, Present, and Future. IntechOpen.

DOI: https://doi.org/10.5772/intechopen.82497.

[11] Taha Gökmen Ülger, Ayse Nur Songur, Onur Çirak FPÇ, 2018. Role of Vegetables in Human Nutrition and Disease Prevention. IntechOpen.

DOI: http://dx.doi.org/10.5772/intechopen.77038.

[12] Elkhalifa, A.E.O., Alshammari, E., Adnan, M., et al., 2021. Okra (Abelmoschus esculentus) as a potential dietary medicine with nutraceutical importance for sustainable health applications. Molecules. 26(3), $1-21$.

DOI: https://doi.org/10.3390/MOLECULES26030696.

[13] Shahidi, F., Yeo, J., 2018. Bioactivities of Phenolics by Focusing on Suppression of Chronic Diseases : A Review. (Figure 1), 1-16.

DOI: https://doi.org/10.3390/ijms19061573.

[14] Islam, M.T., 2019. Phytochemical information and pharmacological activities of Okra (Abelmoschus esculentus): A literature-based review. Phyther Res. 33(1), 72-80.

DOI: https://doi.org/10.1002/ptr.6212.

[15] Yin, G., Wang, Z., Wang, Z., Wang, X., 2018. Topical application of quercetin improves wound healing in pressure ulcer lesions. Experimental Dermatology. 27(7), 779-786.

DOI: https://doi.org/10.1111/exd.13679.

[16] Howe, C.G., Li, Z., Zens, M.S., et al., 2017. Dietary B vitamin intake is associated with lower urinary monomethyl arsenic and oxidative stress marker 15-F2t-isoprostane among New Hampshire adults. J Nutr. 147(12), 2289-2296.

DOI: https://doi.org/10.3945/jn.117.253419.

[17] Lindberg, A., Rahman, M., Persson, L-åke, Vahter, M., 2008. The risk of arsenic induced skin lesions in Bangladeshi men and women is affected by arsenic metabolism and the age at first exposure. 230, 9-16. DOI: https://doi.org/10.1016/j.taap.2008.02.001.

[18] Pierce, B.L., Argos, M., Chen, Y., et al., 2011. Original Contribution Arsenic Exposure, Dietary Patterns, and Skin Lesion Risk in Bangladesh : A Prospective Study. 173(3), 345-354. 
DOI: https://doi.org/10.1093/aje/kwq366.

[19] Działo, M., Mierziak, J., Korzun, U., Preisner, M., Szopa, J., Kulma, A., 2016. The potential of plant phenolics in prevention and therapy of skin disorders. Int J Mol Sci. 17(2), 1-41.

DOI: https://doi.org/10.3390/ijms17020160.

[20] Ombra, M.N., Fratianni, F., Granese, T., 2014. Natural Product Research : Formerly Natural Product Letters In vitro antioxidant, antimicrobial and anti-proliferative activities of purple potato extracts (Solanum tuberosum cv Vitelotte noire ) following simulated gastro-intestinal digestion. (December), 37-41. DOI: https://doi.org/10.1080/14786419.2014.981183.

[21] Jadwiga, A., Id, K., Pobere, J., Przewodowska, A., Id, W.P., Milczarek, D., 2019. Antioxidant properties of potato tubers (Solanum tuberosum L .) as a consequence of genetic potential and growing conditions. Published online. 1-14.

DOI: https://doi.org/10.1371/journal.pone.0222976.

[22] Praveen Kumar, P., Swatantra Bahadur Singh, S.J., 2014. Overview On Anti-Ulcer Activity Of Basella Alba : A Therapeutic Herb. 5(June), 49-61.

[23] Umar, K., Muhammad, M., Sani, N., Muhammad, S., Umar, M., 2015. Comparative Study of Antioxidant Activities of the Leaves and Stem of Ipomoea aquatica Forsk (Water Spinach). Niger J Basic Appl Sci. 23(1), 81 .

DOI: https://doi.org/10.4314/njbas.v23i1.12.

[24] Rosas-Cruz, G.P., Silva-Correa, C.R., Calderón-Peña, A.A., et al., 2020. Wound Healing Activity of an Ointment from Solanum tuberosum L. "Tumbay Yellow Potato" on Mus musculus Balb/c. Pharmacogn J. 12(6), 1268-1275.

DOI: https://doi.org/10.5530/PJ.2020.12.175.

[25] Owumi, S.E., Odunola, O.A., Gbadegesin, M.A., Nulah, K.L., 2013. Protective effect of Juglans nigra on sodium arsenite-induced toxicity in rats. 5(3), 183188.

DOI: https://doi.org/10.4103/0974-8490.112425.

[26] Deshmukh, S.A., Gaikwad, D.K., 2014. A review of the taxonomy, ethnobotany, phytochemistry and pharmacology of Basella alba (Basellaceae). J Appl Pharm Sci. 4(1), 153-165.

DOI: https://doi.org/10.7324/JAPS.2014.40125.

[27] Adhikari, R., Hn, N.K., Sd, S., 2012. Review Article A Review on Medicinal Importance of Basella alba L. 4(2), 110-114.

[28] Yasin, H., Tariq, F., Sameen, A., et al., 2020. Ethanolic extract of okra has a potential gastroprotective effect on acute gastric lesions in Sprague Dawley rats. Food Sci Nutr. 8(12), 6691-6698.
DOI: https://doi.org/10.1002/fsn3.1963.

[29] KIM, Y.H., IDA, M., YAMASHITA, S., et al., 2012. Anti-Allergic Effects of Kakrol (Momordica dioica Roxb.) Flesh Extract. Biosci Microbiota, Food Heal. 31(1), 1-6.

DOI: https://doi.org/10.12938/bmfh.31.1.

[30] Baskaran, G., Salvamani, S., Azlan, A., Ahmad, S.A., Yeap, S.K., Shukor, M.Y., 2015. Hypocholesterolemic and Antiatherosclerotic Potential of Basella alba Leaf Extract in Hypercholesterolemia-Induced Rabbits. Evidence-based Complement Altern Med. DOI: https://doi.org/10.1155/2015/751714.

[31] Dua, T.K., Dewanjee, S., Gangopadhyay, M., Khanra, R., Zia-Ul-Haq, M., De Feo, V., 2015. Ameliorative effect of water spinach, Ipomea aquatica (Convolvulaceae), against experimentally induced arsenic toxicity. J Transl Med. 13(1), 1-17.

DOI: https://doi.org/10.1186/s12967-015-0430-3.

[32] Devi, N., 2017. Medicinal Values of Trichosanthus cucumerina L. (Snake Gourd) - A Review. Br J Pharm Res. 16(5), 1-10.

DOI: https://doi.org/10.9734/bjpr/2017/33575.

[33] Lovat, C., Nassar, A.M.K., Kubow, S., Li, X., 2015. Donnelly DJ. Critical Reviews in Food Science and Nutrition Metabolic Biosynthesis of Potato ( Solanum tuberosum L .) Antioxidants and Implications for Human Health. (March), 37-41.

DOI: https://doi.org/10.1080/10408398.2013.830208.

[34] Rothman, K.J.G.S., 1998. Modern Epidemiology. Second Edition. Philadelphia: Lippincott Williams and Wilkins.

[35] Forman, M., 1998. Nutritional Epidemiology. 2nd ed. (Walter Willett 1998, ed.). Oxford University Press, New York.

DOI: https://doi.org/10.1093/ajen/69.5.1020.

[36] Islam, S.N., Khan, M.N.I., Akhtaruzzaman, M., 2010. Food Composition Tables and Database for Bangladesh with Special Reference to Selected Ethnic Foods. Institute of Nutrition and Food Science, University of Dhaka. fpmu.gov.bd $>$ sites $>$ default $>$ files Finalreport. [Accesed October, 2021]

[37] Septembre-Malaterre, A., Remize, F., Poucheret, P., 2018. Fruits and vegetables, as a source of nutritional compounds and phytochemicals: Changes in bioactive compounds during lactic fermentation. Food Res Int. 104, 86-99.

DOI: https://doi.org/10.1016/j.foodres.2017.09.031.

[38] Tungmunnithum, D., Thongboonyou, A., Pholboon, A., Yangsabai, A., 2018. Flavonoids and Other Phenolic Compounds from Medicinal Plants for Pharmaceutical and Medical Aspects: An Overview. Medi- 
cines. 5(3), 93.

DOI: https://doi.org/10.3390/medicines5030093.

[39] Singh, S.R.S., 2007. Amelioration of arsenic toxicity by L-Ascorbic acid in laboratory rat. J Env Biol. PMID: 17929753.

[40] Ah Kang, M., Choung, S.Y., 2016. Solanum tuberosum L. cv Hongyoung extract inhibits 2,4-dinitrochlorobenzene-induced atopic dermatitis in $\mathrm{NC} /$ Nga mice. Mol Med Rep. 14(4), 3093-3103.

DOI: https://doi.org/10.3892/mmr.2016.5595.

[41] Perchuk, I., Shelenga, T., Gurkina, M., Miroshnichenko, E., 2020. Burlyaeva M. composition of primary and secondary metabolite compounds in seeds and pods of asparagus bean (Vigna unguiculata (L.) Walp.) from China. Molecules. 25(17), 1-16. DOI: https://doi.org/10.3390/molecules25173778.

[42] Avanza, M.V., Álvarez-Rivera, G., Cifuentes, A., Mendiola, J.A., Ibáñez, E., 2021. Phytochemical and functional characterization of phenolic compounds from cowpea (Vigna unguiculata (1.) walp.) obtained by green extraction technologies. Agronomy. 11(1). DOI: https://doi.org/10.3390/agronomy11010162.

[43] Sayeed, I., SatishAjay, S.K., Hegde, K., 2017. Pharmacological activities of Vigna unguiculata ( L )
Walp: A review. Int J Pharma Chem Res. 3(1), 44-49. [44] Moreira-Araújo, R.S.D.R., Sampaio, G.R., Soares, R.A.M., Da Silva, C.P., Araújo, M.A.D.M., Arêas, J.A.G., 2018. Identification and quantification of phenolic compounds and antioxidant activity in cowpeas of brs xiquexique cultivar. Rev Caatinga. 31(1), 209216.

DOI: https://doi.org/10.1590/1983-21252018v31n124rc.

[45] Marsetya, Y.R., Mudjijono, H.S., 2011. Antioxidant activity, phenolic and flavonoid contents of snake gourd (Trichosanthes anguina) extract. Biofarmasi J Nat Prod Biochem. 7(2), 77-86.

[46] Durazzo, A., Lucarini, M., Novellino, E., Souto, E.B., Daliu, P., Santini, A. Beneficial Properties - Focused on Antidiabetic Role - For Sustainable Health Applications.

DOI: https://doi.org/10.3390/molecules24010038.

[47] Xu, D.S., Qin, L.Y., Han, M.L.Q., 2019. Physicochemical properties, phenolic profiles, antioxidant capacities, and inhibitory effects on digestive enzymes of okra (Abelmoschus esculentus ) fruit at different maturation stages. 56, 1275-1286.

DOI: https://doi.org/10.1007/s13197-019-03592-1. 